\title{
Energy Attacks on Mobile Devices
}

\author{
Ashish Kundu \\ IBM T J Watson Research \\ Yorktown Heights, NY \\ akundu@us.ibm.com
}

\author{
Zhiqiang Lin \\ University Texas at Dallas \\ Dallas, USA \\ zhiqiang.lin@utdallas.edu
}

\author{
Joshua Hammond \\ University Texas at Dallas \\ Dallas, USA
}

\begin{abstract}
All mobile devices are energy-constrained. They use batteries that allows using the device for a limited amount of time. In general, energy attacks on mobile devices are denial of service (DoS) type of attacks. While previous studies have analyzed the energy attacks in servers, no existing work has analyzed the energy attacks on mobile devices. As such, in this paper, we present the first systematic study on how to exploit the energy attacks on smartphones. In particular, we explore energy attacks from the following aspect: hardware components, software resources, and network communications through the design and implementation of concrete malicious apps, and malicious web pages. We quantitatively show how quickly we can drain the battery through each individual attack, as well as their combinations. Finally, we believe energy exploit will be a practical attack vector and mobile users should be aware of this type of attacks.
\end{abstract}

\section{INTRODUCTION}

Smart mobile devices are everywhere: iPhones, Android phones, Windows phones, Blackberry, iPads, Android tablets, Smart watches, Google glasses, so on and so forth. The computing power of these devices is on a steep increase day-by-day with multi-core devices already on the market such as iPhone $4 \mathrm{~S}$ and iPhone 5 . The power of these devices and what they offer is being enabled by millions of apps, readily available and inexpensive for the majority of users. With such a thriving ecosystem, the security threats that come with these devices and apps are also on a steep rise. Android app stores have been found to contain mal-apps, re-packaged apps that include malwares [15]. Recently, Apple's messaging system iMessage for their iOS devices were compromised and DoS attacks on some devices using iMessage were initiated [8]. It may not be imprudent to say that "security issues are abundant in an ecosystem that enables freedom of sharing, and growth for its producers and consumers". There is a plethora of works that have studied security of smart mobile devices (e.g., [15]).

\footnotetext{
${ }^{*}$ The author contributed to this work and paper during the first half of 2013 when he was a student at Univ. of Texas at Dallas.
}

Permission to make digital or hard copies of all or part of this work for personal or classroom use is granted without fee provided that copies are not made or distributed for profit or commercial advantage and that copies bear this notice and the full citation on the first page. To copy otherwise, to republish, to post on servers or to redistribute to lists, requires prior specific permission and/or a fee.

Copyright 20XX ACM X-XXXXX-XX-X/XX/XX ...\$15.00.
A common aspect of all such devices is they are energy-constrained, unlike their desktop and server counterparts. In particular, these devices include a battery that powers the computation and all its usage. If the battery is drained completely or to an unusable level, neither the user nor the apps installed on it can use the device in the way(s) they need to. What if an attacker manages to carry out attacks that drain the energy of a device so that the device is impaired and cannot be used until it is recharged again? In this paper, we focus on this type of attacks, which we refer to as "energy attacks on smartphones." There have been previous works related to energy attacks on server systems by Wu et al. [13]. Also, there have been works focusing on energy estimation of Android usage [10 12]. However, existing works have not focused on how attackers can carry out energy attacks.

Consider the following use-case of carrying out such an attack - an espionage scenario (relevant to a Bond or a Bourne Identity movie) - "To kill a device": a spy is waiting for a directive from her boss on her smartphone. The directive can arrive in any way - as a call, a message and at any point in next one hour of train ride. The mafia, who want to prevent the spy from carrying out the directive, have to succeed. One way to enable that is to try to kill the spy, a high risk-high return proposition. The other way to enable that is to "kill the device" via energy attacks. The mafia uses its pre-created hundreds of accounts on google voice, and makes calls to this device continuously from these google voice accounts. The spy cannot shutdown her device, or disable calling/messaging capabilities because she has to receive the directive via the smartphone. The continuous calls lead to "vibration" and/or ringing of the device, leading to energy drainage, and eventually "killing the device" by draining most of its energy.

Such an attack can be easily thwarted by enabling a white list of who can call and who cannot call. However, if an app used by the spy has such a vulnerability or has been developed with a backdoor to carrying out energy attacks - such an access control would not work. The question is how effective such app-based attacks would be? This paper delves deeper and studies the different components of Android operating system, its energy usage and how fast battery can be drained on Android devices. Android commands the smartphone industry as the most-used operating system, as of now. An attacker could potentially include malicious code into an otherwise benign application in an attempt to deny access to a user. Even advanced users may be unaware of how much battery power each component can drain, and how quickly could a malicious application could drain the battery. Through our experiments and analysis we seek to understand the elements of a successful energy attack.

Our contributions: We present a taxonomy of the energy attacks on mobile devices $(\$ 2$, and use it to guide the development of en- 
ergy exploits ( \$3 by systematically exploring hardware, software, and network elements $(\$ 4)$. Our experimental result $(\$ 5$ shows that our energy attacks are practical, and can drain battery in a few minutes with a combination of multiple exploits.

\section{TAXONOMY OF ENERGY ATTACKS}

In this section, we first present the principles behind energy attacks in $\$ 2.1$, then describe why energy attacks are useful with several compelling use cases in $\$ 2.2$ and the delivery modesl for energy attacks in $\$ 2.3$ and finally present the taxonomy of such attacks including the elements of on how to carry out energy attacks and how to construct the exploits in $\$ 2.4$

\subsection{Principle of Energy Attacks}

Energy attack is carried out as and when any consumption and/or depletion of energy on a device is effected by processing of operations with no legitimate purposes.

Let $\mathcal{A}$ be the set of operations that processing of operations and $D$ be a device. Suppose $\alpha \in \mathcal{A}$ on $D$ leads to depletion of energy level by $\delta>0$ amount, then $\alpha$ is a candidate operation for carrying out energy attacks. An energy-attack comprises of carrying out one or more candidate operations on the target device with an objective to deplete the energy of a device by an amount that is greater than zero and less than or equal to $100 \%$. If the event that triggers processing of a subset of candidate operations drawn from $\mathcal{A}$ on $D$, has its origin in a malicious intention, then the process of energy depletion on the device is called an energy attack. If the event does not have its origin in a malicious intention, then it could be called a bug.

In current computational model, we can safely assume that all operations are candidate operations including the "NOP" operations implemented at the hardware level. "NOP" operations do not lead to any operation being carried out, but it definitely consumes at least one CPU cycle, and thus leads to consumption of energy, which would lead to depletion of energy on the device (theoretically carrying out some number of NOP operations is a form of energy attacks.

A practical energy attack would associate a temporal constraint on the amount of energy being depleted: the rate of consumption of energy by a specific combination of the candidate operations is defined as the ratio of amount of energy consumed over a period of $t_{\delta}$ time and $t_{\delta}$. The greater the rate of consumption of energy, the more the efficacy of the combination in terms of energy attack.

\subsection{Malicious Motivation and Use-cases}

The question is why is an "energy attack on devices" an appealing attack, and what would an attacker gain from carrying out such an attack? Let us describe some other use-cases in addition to the espionage one described in $\S 1$.

- To kill the devices that are parts of a botnet: In order to control the extent of damage a network of device-based bots can carry out, the immediate mitigation maybe to shut those devices down or remove them from the network (as is done in the case of desktops and servers). However, unlike the desktops and servers, devices are mobile, move from one network to another, from $4 \mathrm{G}$ to $\mathrm{WiFi}$ and thus it is harder to control them. Therefore one feasible option is to "drain the energy of those devices remotely" by carrying out energy attacks.

- If law-enforcement gets to know the devices being used by terrorists posing an imminent threat of a terrorist attack, in order to disband their strategy and prevent them from communicating with each other, a stealth energy attack could be launched on those devices by law-enforcement.

- Mobile devices are being used to detonate bombs. If lawenforcement know the mobile devices that are attached to the bomb, or being used to detonate the bombs, perhaps a way to prevent the bomb from being detonated is to drain the device of energy.

- Protection of a personal device after being lost or stolen: it is common to lose or get one's device stolen. Devices contain sensitive personal and corporate data; many of the smart devices today have active sessions with web services such as gmail, facebook; the devices are configured to ask for a password at startup. The owner of the device would want to minimize the risk of the device being on the hands of a malicious user X. By carrying out an energy attack on the device, the owner would accomplish some or all of the following: (1) prevent the user $\mathrm{X}$ to gain access to the device at all as a restart would ask for a password, (2) prevent the user $\mathrm{X}$ to gain access to the device at least for sometime until it is charged again, (3) prevent the user to gain access to the active sessions, which are lost after the device re-starts.

- Business competitors carry out stealthy attacks: Consider that one of the businesses in the smartphone market wants to show that its battery is more powerful than the a competitor. In order to bolster its claim among users of the competitor's device, it could launch energy attacks remotely with an objective to drain the energy quicker than it does for common user activities. That would demonstrate the battery life and convince the users that the battery of the competitor's device is not up to the expectation.

- A futuristic scenario: As vehicles involve computing and smart-networks as part of enabling vehicular networks, they would also rely on battery power to remain operable when not being driven. For example, in order to prevent someone from traveling, one may need to jeopardize their car. If the car is not within physical control, can someone attack its battery by sending multiple network packets or unlocking requests, or locking requests? Once the battery dies, perhaps that would make the vehicle, which is highly reliant on the battery, remain nonoperational until the battery is recharged again.

\subsection{Delivery Models of Energy Attacks}

Attackers can make use of the following delivery and business models:

- Energy-Attacks-as-a-Service: As a cyber-underworld proposition or available to common users, such a service would allow attackers to carry out targeted energy attacks for monetary payments or similar other services as exchange. For legitimate usage, such a service would require a requesting party to verify its identity against the device (that it owns or has authoritative power) on the device, or it has a court order to do so, or certain government agencies have provided the power to carry out such an attack. As a business model, the attackers can use a subscription-based business model, or pay-per-use.

- Back-doors for efficient energy attacks: Devices can be designed with backdoors available to legitimate parties for energy attacks. The legitimate parties could be the owner, network providers, vendor or government agencies. However, 


\begin{tabular}{|c|c|c|c|c|c|c|c|}
\hline Goal & Targets & Control & $\begin{array}{l}\text { Location of } \\
\text { Launch }\end{array}$ & Elements & $\begin{array}{l}\text { Degree of } \\
\text { Stealthiness }\end{array}$ & User Control & Process \\
\hline Kill device & $\begin{array}{l}\text { Targeted } \\
\text { •One device } \\
\text { •Multiple devices } \\
\qquad \text { Constraint- } \\
\text { satisfying } \\
\cdot \text { All/any }\end{array}$ & Controlled & $\begin{array}{l}\text { Local: } \\
\text {-App-driven } \\
\text {-System-driven }\end{array}$ & $\begin{array}{l}\text { Hardware } \\
\text {-Resources } \\
\text {-Operations }\end{array}$ & $\begin{array}{l}\text { Least stealth } \\
\text { (detected by } \\
\text { onlookers) }\end{array}$ & Detectability & $\begin{array}{l}\text { Attacker } \\
\text { devices }\end{array}$ \\
\hline $\begin{array}{l}\text { Partially } \\
\text { drain }\end{array}$ & $\begin{array}{l}\text { Un-targated } \\
\text { - Constraint-satisfying } \\
\text { devices } \\
\text {-All/any }\end{array}$ & $\begin{array}{l}\text { Un- } \\
\text { controlled }\end{array}$ & $\begin{array}{l}\text { Proximity } \\
\text { •Bluetooth }\end{array}$ & $\begin{array}{l}\text { Software } \\
\text {-Resources } \\
\text {-APIs }\end{array}$ & $\begin{array}{l}\text { Stealthy from } \\
\text { onlookers } \\
\text { (detected by } \\
\text { user) }\end{array}$ & $\begin{array}{l}\text { Mitigation } \\
\text {-Shutdown } \\
\text { device } \\
\text { •Stop } \\
\text { apps/services }\end{array}$ & $\begin{array}{l}\text { Human } \\
\text { involvement }\end{array}$ \\
\hline $\begin{array}{l}\text { Event-based } \\
\text { drain }\end{array}$ & & & $\begin{array}{l}\text { Remote } \\
\text { •Network- } \\
\text { driven }\end{array}$ & $\begin{array}{l}\text { Network } \\
\text { - Resources } \\
\text {-Network } \\
\text { operations } \\
\text {-Data transfer }\end{array}$ & $\begin{array}{l}\text { Stealthy from } \\
\text { user } \\
\text { (detected by } \\
\text { system app) }\end{array}$ & & $\begin{array}{l}\text { Automated } \\
\text { agents }\end{array}$ \\
\hline \multirow[t]{4}{*}{$\begin{array}{l}\text { Ruin battery } \\
\text { performance }\end{array}$} & & & $\begin{array}{l}\text { Hybrid: mix of } \\
\text {-Local } \\
\text {-Proximity } \\
\text {-Remote }\end{array}$ & & $\begin{array}{l}\text { Stealthy from } \\
\text { system app } \\
\text { (detected by } \\
\text { third-party } \\
\text { app) }\end{array}$ & & Stealth mode \\
\hline & & & & & $\begin{array}{l}\text { Stealthy from } \\
\text { apps } \\
\text { (detected } \\
\text { from system } \\
\text { logs) }\end{array}$ & & Attack policies \\
\hline & & & & & & & $\begin{array}{l}\text { Event-based } \\
\text { progress }\end{array}$ \\
\hline & & & & & & & Workflow \\
\hline
\end{tabular}

Figure 1: Taxonomy of energy attacks

such backdoors need to be enabled with stringent access control in order to prevent exploitation by malicious attackers.

Some concerns against energy attacks: Energy attacks could be ineffective when the device is being charged. Moreover, even if there is value that can be gained out of "killing one's device" via energy attacks, how effective would be carrying out such attacks? How much time would it require for an attacker to drain the energy of a device with the available exploits? What information is essential for the attacker to know about the real-time status of the device in order to make the attack successful?

\subsection{Taxonomy}

In this section, we present the taxonomy of energy attacks. The following Figure 1 specifies the taxonomy, and the different technical aspects of the attack - why and how. Each column describes the attack in a more detailed manner.

Energy attacks are classified with respect to the following parameters:

1. Goals: There could be four different goals depending how much energy is drained. The first one is to completely drain the energy of the device (kill the device). The second one is to drain the energy partially by a certain percentage or by certain rate or by some specific amount. The third goal is to start and/or stop draining the energy of a device based on some events, and the last one is to drain the energy of the battery so that the battery performance is ruined over a period of time.

2. Targets: Targets of the attack can be one specific device or more than one devices, belonging to one individual or group or satisfying some constraints, such as location. The attack could be un-targeted - any device that satisfies a constraint such as having an app or visiting a webpage can be attacked.

3. Control: "Is the attack controlled by the attacker or uncontrolled" is an important aspect of the attack. Whether the attack can start or stop an attack and select or de-select some targets at the attacker's will defines whether it is controlled or not.

4. Locations of launch: Where is the attack being launched from - where is the attacker? The attack is definitely occurring on the device, but it may be launched from an app or webpage on the device. It maybe launched from other devices in close proximity, or remotely over the network.

5. Elements of the attack: In order to build an exploit, several elements need to be used together. Hardware resources such as GPS, sensors and their operations could be used. Software resources such as system calls, API, memory allocation and de-allocation, locking and unlocking can be used for building an attack. Network-level operations such as data transfer, 
control operations such as handshaking protocols and various network resources such as the bandwidth and antenna could be used to build energy attacks.

6. Process of the attack: An exploit is built, but how to actually implement the attack on a specific target in a controlled or un-controlled manner? Devices could act as attack origins; humans may be involved in launching and controlling the attack; automated agents could be involved. The attack could be delivered in stealth mode so that the user could not discern whether there is an attack going on or it is the common behavior of the device battery; what are the attack policies - how, when and from where to deliver the attack, and how to monitor and manage the attack - based on what events? A workflow may be used to describe an attack delivery. For example, an attack is delivered by sending a message to the user about a free app, and after the user installs it, the app then talks to its command center on an attacker device on how to start the attack and how to monitor such an attack.

Degree of Stealthiness To determine whether an attack is stealthy or not stealthy at all, we have defined a scale of detectability of energy attacks (by detecting use of some components):

1. Detectable from a distance (stealthiness: 0): a component could be detected to be used if the device were, for example, in the user's pocket or purse.

2. Detectable during normal usage (stealthiness: 1): includes things like a noticeable change in responsiveness of the device.

3. Detectable with built-in applications (stealthiness: 2): the device comes standard with some program or monitor that detects high usage of a component.

4. Detectable with third party applications (stealthiness: 3 ): a component's usage is easily detectable with applications available through the standard marketplace.

5. Detectable with access to low level functions or system access (stealthiness: 4): to detect a component the user may have to write a program to read and analyze some measurements, or may have to access system logs or process information in order to find which component is using significant battery power.

For the first two levels, we would expect a component's usage to be detected by almost every Android user. To detect attacks that are at the third and fourth level, the user needs to have certain minimal knowledge of the device - such as which applications could be used to monitor CPU usage and battery drainage. The user needs to realize that the battery is being drained more than usual, and needs to know where to look for these applications and how to use them. At the fifth level we would only expect a user to detect the drain if the user has some familiarity with development on the Android platform. At the fifth level, a user would likely need to actively monitor battery usage and then need the knowledge and experience necessary to write a new application to detect it, or to access system resources to determine the cause.

We decided that this would be the toughest level as we do not foresee any component being completely unnoticeable or even requiring hardware access to monitor and detect. Because each level requires more user involvement and skills on the part of the user from 1 to 5 in that order, we use only the lowest level for each component. This will give an idea of the very minimum amount of knowledge and skill required to detect the use of a component that has a large impact on the battery. For this part of our testing we used our application to turn on each component but did not set a certain battery level threshold. While each component is running we attempt to detect that the particular component is running either at an abnormal level, or running when there should not be an application using that component ( other than our own). We start by attempting to detect it at level one. If significant usage is detected we stop and record the level at which the usage was detected. If, after a reasonable effort, the usage is not detected, we go up to the next step and again attempt to detect the component's usage. For the purpose of this research we do not attempt to find which application is using the component, but merely that the component is being used in a way that may drain the battery. Finally we give information on each component that may make use of the component more difficult or preventable. We specifically make note of settings that may need to be on for a component to work. This is closely coupled with the permissions required for an application to make use of each component. Knowing this makes it much easier to detect an application that may have a large impact on battery before it is installed. This information allows users to make a more informed decision about whether an application can use the access rights, that the users gives to it, to drain the battery.

\section{BUILDING AN EXPLOIT}

Building an exploit for energy attack does not depend on traditional vulnerabilties in software stack, hardware devices or in cryptography. That makes it easy to build such an attack and hard to defend.

An exploit uses each of the aspects of the taxonomy discussed earlier. An attacker decides the goal(s), the targets of the attack and controls on the attack. Based on the available methods of attack delivery, the attacker can decide the elements to be used for the attack. If the attack delivery method available to the attacker is via installing an app on the device via a promotional ad, the attacker then goes ahead and implements the app that uses several elements of the attack. If the attacker has access to a backdoor on the device, then based on the elements exposed via the backdoor, exploit can be built.

Delivering the attack after building an exploit can be implemented in several ways. By getting the user to install the app by luring it for a coupon, or by some other social engineering method, the attack via the app can be delivered. The delivery model then can be Energy-attack-as-a-Service or via backdoor exposed via the app. How to get the attack delivered is part of building the attack; however, due to constraints on the page length, we would not go into further details.

Delivering the exploit to the device Several techniques can be used to deliver the exploit to the device(s). Some of the ways to attack a device are:

Attacker knows the phone number:

1. Attacker sends a message with a coupon or similar monetary incentive and asks the user to install the app or visit website to get the coupon.

2. App: delivers more control on the attack. An app could include the exploits during its development, or introduced during the lifecycle.

3. Visiting the website delivers the attack only for the time of visit. 
Attacker knows the user's name:

1. If the attacker is a social network or one of its admins or a gaming service that the user has enrolled in, and has its app installed on the device, or if the attacker can carry out a network-based man-in-the-middle attack by injecting traffic:

2. the attacker pushes data, scripts and events to trigger the app.

3. the attacker then can lure the user as in previous example to install other apps or visit webpages.

Attacker knows the webpages the user visits:

1. The attacker controls the webpages, then it can add energyconsuming software and network elements. In particular, a web-page may contain java-scripts that carry out energy attacks. These java-scripts could contain infinite loops, or recursive code, or data download, malicious applets, etc., to drain the battery.

2. The attacker otherwise can use Google Ads or such other services for displaying advertisements including javascripts that do not look malicious but carry out energy attacks.

\section{ELEMENTS OF ENERGY ATTACKS}

In this section, we describe the elements of the exploit. There are in general three types of elements: hardware, software, and network. Hardware elements are rarely exposed as they are - software elements are almost always combined with hardware elements. For example, accessing GPS is allowed from an app, which thus can include some software-based elements in the exploit. Network-based elements can be used along with the software elements. A software app can maliciously download more data than needed thus consuming the energy via network elements and software elements. In order to determine how effective each hardware, software, and network element is towards carrying out energy attacks, we carried out several experiments by developing the exploits as apps, webpages based each elements or a combination of them. A high level overview of these elements is presented in Fig.2 In the following, we present how to construct the concrete attacks by using these elements.

Experiment Setup In order to determine the efficacy of energy attacks by using the components, we need to determine how long it takes to achieve the goal of the attack - draining energy by using the corresponding components. We carried out two types of experiments: (1) for draining energy from 100\% to 0\% (kill the device), and (2) an energy depletion by 5\% (partial draining). We also did a control test. In each of our tests the screen is wake-locked, which means the display will not turn off and the phone will not enter sleep mode. This is necessary so that the operating system does not begin powering down components and give inaccurate readings as to their battery usage. Thereby we also acquire the wake-lock for the control test. By using this wake-locked control we can see how much energy is consumed by each component compared to the wake-lock and that should give us an accurate comparison of which components consume more energy. $\$ 5$ will present the detailed experimental results.

Single vs. Combination: Exploits could be based on a single element or a combination of elements, and can be parallel or sequential. Parallel exploits are expected to be more effective in carrying out energy attacks because, the software, hardware or network elements each thread or parallel process uses consumes power, as well
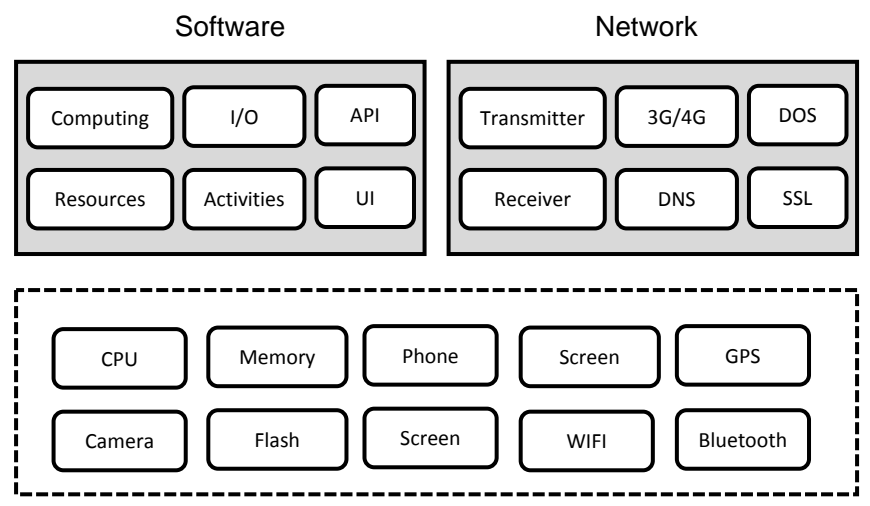

Hardware

Figure 2: Overview of the Exploit Elements

as there is a cost of scheduling and bookkeeping of threads/processes at the system level. In the exploits we have experimented with, if it is a combination of elements, then we have tried to maximize the battery drainage by making it parallel - either at process-level or at the app level.

While most energy attacks would be a complex combination of elements of different categories - software, hardware, network and would be used both in sequential and parallel; in order to quantify how effective such exploits would be, we need to understand how effective and quantify the energy draining capabilities of exploits developed based on single elements.

\subsection{Hardware Elements}

Using hardware components of a device can drain the battery significantly. Several hardware components/sensors are part of the hardware packaging of the device. Some such components covering some of the common components across devices running Android and iOS: Other than CPU, display and brightness via ambient sensor, camera, flash, bluetooth, Wifi, 3G or 4G, GPS, gyroscope, accelerometer, proximity sensor, and magnetometer.

The fundamental method of carrying out effective hardware-level energy attack is: to develop an exploit based on the hardware elements that can be used concurrently with minimum authorization needed, and consume most amount of power when used concurrently together. Determine these elements and their combination statically and/or dynamically depending on the state of the device and the elements.

\subsubsection{Basic Components}

$C P U$ : In order to determine how effective CPU-intensive exploits would be, we used an app that carries out matrix multiplication using two threads. The matrix multiplication starts with two initial matrices and then continues to multiply the resulting matrix by one of the starting matrices. In our experiments, this has a CPU utilization of up to $90 \%$, which is a good measure of the efficacy of CPU-intensive exploits. Compute-intensive exploits may or may not be stealthy at all depending on how the system handles high CPU utilization - some systems may not respond to user interaction well, which makes the attack somewhat less stealthy.

Memory: Memory, especially video memory consumes a large amount of energy. Playing video would exploit this consumption of energy, which however, would not be stealthy at all. In our implementation, we loaded a video to the memory, and played it in an infinite loop. However, playing different videos would be more 
effective as it includes writing to the video memory for each new video other than reading and refreshing the memory.

Phone: In order to exploit the phone call functionality, an echo number is used as the other number to be called. The advantages of using an echo number are: it does not require extra participants to receive a call; it does not disconnect prematurely. It can be stealthy until the user tries to use the phone functionality and discovers that a call is being made (phones do not have the mechanism for multiple active call sessions).

Camera flash: Camera flash can be used to attack the device, but it is not stealthy at all. In our exploit, we have an infinite loop that turns the camera's flash on then off. Theoretically, leaving the flash light on will drain less energy than when turned off and on continuously.

Camera: Camera of a device uses several resources and thus an important candidate for building ane exploit. This element is of medium stealth, but user interaction with the camera would reveal the app being used. To exploit the photo capability and we used the camera interface, rather than the normal method of calling another application to take care of the actual photography. This gave us more control over what components were being used, allowed us to take pictures rapidly, and allowed us to automate the process.

\subsubsection{Sensors}

There are several other sensors that can be used to build exploits. Android supports a sensor API framework, which allows the program to determine the power requirements of each sensor. An exploit may use these APIs and determine the sensors that require the most power dynamically and start using some of them. In the following discussion, we describe some of the sensors with which we have developed exploits. Other sensors such as accelerometer, gyroscope, proximity sensors, light sensors, magnetometer, temperature and pressure sensors can be exploited similarly. Adding a listener, removing a listener, probing a sensor, acquire the sensor data continuously are different actions that can be carried out repetitively and in parallel to drain the energy.

Screen brightness sensors: Screen brightness is highly non-stealthy and also consumes large amount of energy. For testing screen brightness we turned the brightness up to its maximum while in our wake locked state. It is important to note that this test would not be accurate without the wake-lock that keeps the display active. For this component to drain the battery at the levels we record in our experiment, the display must remain active throughout the entire period. It is also important to note that during our experiments we were unable to turn up the brightness when the battery was low (as controlled by the Android OS). This also requires that the phone stay awake and may not functionally drain the battery from the background.

GPS: GPS component as an element of energy attacks can be effective and are highly non-stealthy, as most devices show use of GPS on the top-bar of the phone. To test the GPS component, we enabled a location listener and constantly read the location of the device. This is similar to how a GPS would have to be used with a map application, to maintain a current and accurate location.

Bluetooth: Switching bluetooth on and off itself can consume energy. This activity can be a stealthy one as long as bluetooth activity can be hidden from the display and UI.

\subsection{Software Elements}

Software elements may be compute-intensive, I/O-intensive, networkintensive or sensor-intensive, or a mix of both of them. The softwarelevel elements include OS-level APIs, resources such as locks and semaphores, threads/processes, libraries, software cache, file sys- tem, system calls, sockets, events and notifications, and messages. Higher-level elements are activities, UI components, Java libraries and so on. We have developed exploits on a combination of some of these APIs.

In order to develop an effective energy attack based on softwarebased elements, a rule of thumb is: An effective energy attack uses software elements that can be used concurrently and that consume most amount of energy when used together.

Some of the rules of thumbs is to use elements that:

1. lead to as many context switches, memory swapping and I/O as possible.

2. run stealthily - in the background and does not interfere with useability of the device.

3. force using system calls and/or lead to interrupts

4. are complex mathematical operations such as cryptography, matrix multiplication, graphics.

5. belong to different libraries, use data blocks from different memory pages thus defeating the locality-of-reference strategy.

6. start servers that listen to on some ports creating backdoors to the device.

In our experiments, we developed exploits based on cryptography, matrix multiplication, notification, database operations and web-based operations.

Cryptography: Cryptographic operations such as encryption, digital signatures involve complex mathematics and are expensive operations. They also run quite stealthily and are known to consume lot of energy especially when cryptographic accelerators are not in use. Our exploits used RSA encryption and signature generation.

Database Operations: Database I/O also is a stealth operation and is expensive. Table creation, deletion, data addition and removal are to be included in the operations. If caching can be disabled by the attacker programmatically with no higher privilege required, it should be disabled.

Notifications: Notifications are very common in consumer applications and we thought it would be important to show their impact. To test the screen rotation we constantly swapped between landscape and portrait layouts. Though this component was tested through API calls, we believe that it is also a reasonable estimate for how the device would drain power when the device is rotated to change screen orientation. For our experiment, we constantly opened and closed a notification.

\subsection{Network Elements}

The network elements use the transmitters and receivers of the device - both for $4 \mathrm{G}$, Wifi and bluetooth capabilities. Network operations also lead to use of network stack, use of cryptography when SSL is enabled.

The general principle of development of exploits for energy attacks via network elements is: Use the network operations such that they can be carried out concurrently with maximum data transfer such as wifi and bluetooth used concurrently, as much processing as possible by the network components of the device, synchronize the operations such that the device has to power on and off its transmitters and receivers as much as possible, and inject energy attack payloads when the network communication is vulnerable.

There are several elements related to network and communication that can be exploited: bluetooth-based communication, wifi 
and 4g-based communication, data transfer, control protocols, DNS query attacks and so on.

Bluetooth-based Communication: Switching bluetooth on and off itself can consume energy. This activity can be a stealthy one as long as bluetooth activity can be hidden from the display and UI.

Wifi-download: For our download test, we have a statically coded address for an image on the web. This image is constantly downloaded and written to the SD-card. Though we could have skipped over writing to the SD-card it seems that a common application would not download something to discard it and would likely constantly store downloaded data for the application.

DNS query attack: DNS queries are sent out by the device in clear, and a form of Kaminsky attack can be used to drain the energy of the battery significantly. Moreover, the DNS cache poisoning can be used to make the device send the data several times, send data to hosts from where the destination is unreachable.

DoS Attack: Carry out DoS attacks on the open ports on the device such as via $\mathrm{SYN}$-flodding attacks.

\section{EVALUATION}

In this section, we have described several experiments that we have carried out to determine effectiveness of several hardware, software and network elements in carrying out energy attacks. The experiments include exploits developed based on single elements or a combination of these elements.

Experimental Setup: For our experiments we used a Samsung Captivate Glide (SAMSUNG-SGH-I927) smart phone with Android Gingerbread (Android version 2.3.6). The hardware capabilities covered are: CPU, vibration, camera flash, WiFi download, bluetooth, phone, 4G download, brightness, video playback (video memory), GPS, screen rotation, and camera.

Process of experiments: For each test, the app implementing an exploit we developed, allows the tester to select which components to enable for the test. Then, when the start button is pressed, the application starts each individual component. After every component has been started the application records the current time and takes an initial reading of the battery level (reported in percent). The main application then sleeps for two second intervals while the component runs. Each time it wakes, it checks the current battery level. If the difference between the original battery level and the current level is below the threshold of five percent, then the application goes back to sleep, with the components still running in the background. If the threshold has been met then the application records the current time, compares it with the starting time and makes a notification to the user about the number of minutes the application took to drain to the threshold. For our experiments, we tested each component ten times. This allows us to take an average that compensates for some of the fluctuations we may see in each individual test.

Most effective exploits: Top three exploits that drain the battery from $100 \%$ charge to $0 \%$ and that drain $5 \%$ of the battery with lowest time required are described in the following table. The web attack that includes an exploit delivered by a webpage took two hours and forty five minutes and is the second most effective attack, which moreover can be carried out in a stealth manner. The other two attacks are not stealth but the most effective attack can drain the battery within one hour and forty five minutes.

\begin{tabular}{|l|l|}
\hline Top 3 exploits & $\begin{array}{l}\text { Time in mins } \\
\text { for 100\% draining }\end{array}$ \\
\hline 1. Brightness, CPU and Camera Flash & 104 \\
\hline 2. Web attack & 164 \\
\hline 3. Screen Brightness & 204 \\
\hline \hline Top 3 exploits & $\begin{array}{l}\text { Time in mins } \\
\text { for 5\% draining }\end{array}$ \\
\hline 1. Brightness, CPU and Camera Flash & 4.8 \\
\hline 2. Web attack & 6.5 \\
\hline 3. Brightness, CPU and Camera Flash & $\begin{array}{l}7.2 \\
\text { while charging }\end{array}$ \\
\hline
\end{tabular}

In the following table, we have presented the results of our experiments on the effectives of exploits. For each component we give five statistics: Average teim to drain 5 percent, standard deviation of draining time, maximum time to drain 5 percent, and minimum time. Each measurement of time is recorded in minutes.

\begin{tabular}{|l|l|l|l|l|}
\hline Component & Average & St. Dev. & Max. & Min. \\
\hline Vibration & 19.4 & 1.075 & 21 & 18 \\
\hline CPU & 9.5 & 0.972 & 11 & 8 \\
\hline Camera Flash & 9.3 & 1.059 & 12 & 8 \\
\hline WiFi Down. & 23.5 & 3.598 & 29 & 16 \\
\hline Bluetooth & 25.2 & 5.514 & 36 & 18 \\
\hline Phone & 13.8 & 1.932 & 18 & 12 \\
\hline 4G Down. & 11.1 & 1.197 & 13 & 9 \\
\hline Brightness & 7.4 & 1.075 & 10 & 6 \\
\hline Video & 16.8 & 1.989 & 22 & 15 \\
\hline GPS & 17.4 & 1.734 & 19 & 15 \\
\hline Notification & 26.6 & 4.351 & 33 & 20 \\
\hline Rotation & 17 & 3.197 & 23 & 13 \\
\hline Photo & 12 & 1.764 & 14 & 9 \\
\hline Encryption & 12.3 & 1.059 & 14 & 11 \\
\hline
\end{tabular}

\subsection{Hardware elements}

Our results show that the screen brightness drains battery faster than any other component that we tested. This is closely followed by the camera flash and CPU usage. Using only the brightness would drain the battery in under two and a half hours. For our case of normal usage, this is a rather short amount of time. A smart phone is reasonably expected to last at least 16 hours per day (the time a person would be awake with 8 hours of sleep each day). This suggests that being able to detect and monitor these components could be very important for normal users. For the case of an attack this may be longer than an attacker would like. If the attacker was attempting to close off usage of the phone for a specific time or purpose, this may be ample time to detect the usage, or at least notice the drain and begin charging the phone. We also tested the exploit while the phone was being charged, and we found that energy attacks indeed can succeed while the phone is being charged.

The full drain using screen brightness attack took just over 204 minutes (Figure 3(a). In figure ??, we see a large spike at the end of this graph for the last few percent of the battery life which helps account for the longer than expected drain time. This is where Android automatically lowers the screen brightness to preserve battery life. At that point we have already drained most of the battery and any extended usage would not be possible. This drain may take much longer than an attacker would like and suggests that an attacker would likely need to use multiple components.

To get an idea of how multiple components perform together we tested the three top components - brightness, camera flash, and $\mathrm{CPU}$ at once. If they were completely independent we would ex- 


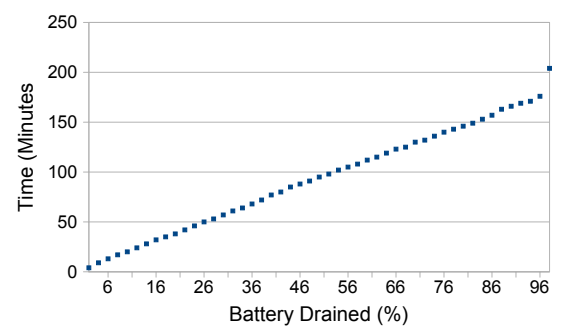

(a) Energy drain of screen brightness attack over full battery life

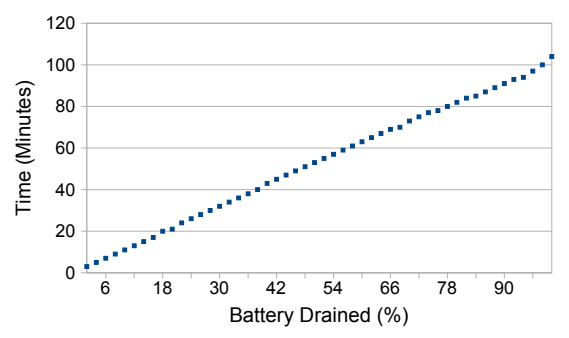

(b) Energy drain of 3 component (brightness, flash and CPU) attack over full battery life

Figure 3: Exploitation using hardware elements.

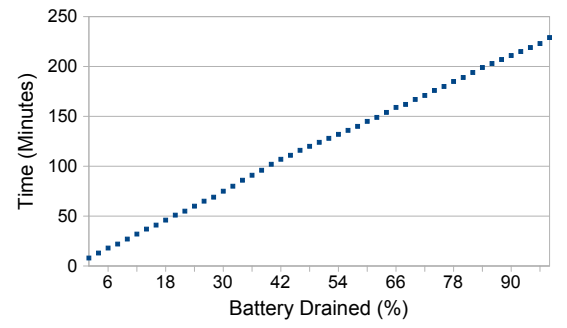

(a) Energy drain of encryption attack over full battery life

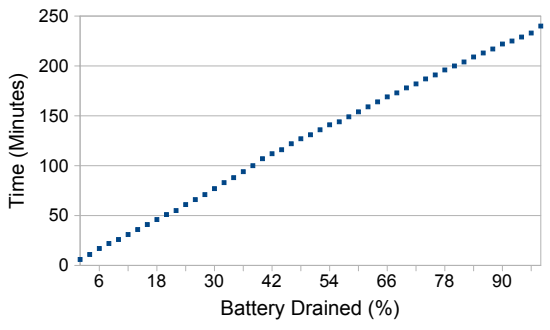

(d) Energy drain of database data encryption, insertion and deletion attack over full battery life

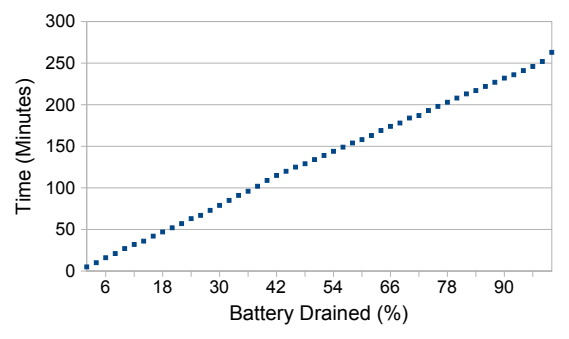

(b) Energy drain of database data generation, addition and deletion attack over full battery life

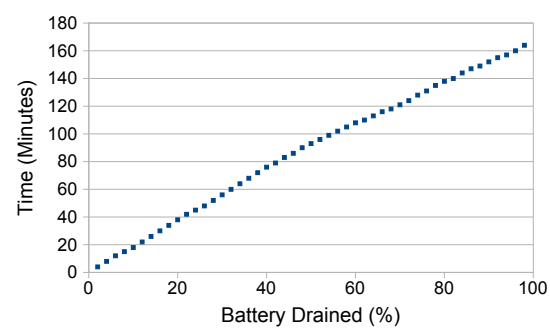

(e) Energy drain of web app attack over full battery life

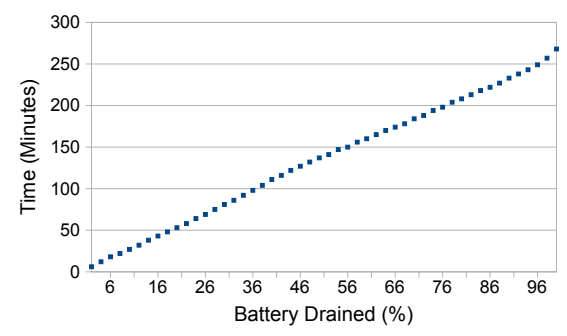

(c) Energy drain of camera attack over full battery life

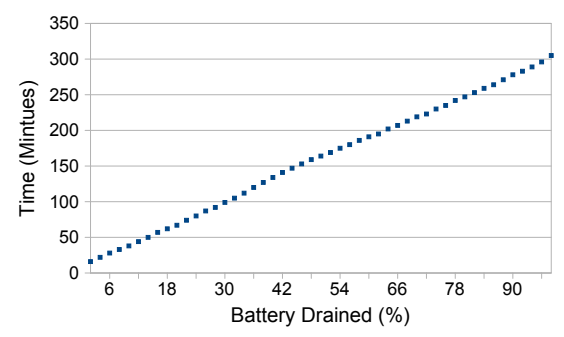

(c) Energy drain of database table addition and deletion attack over full battery life

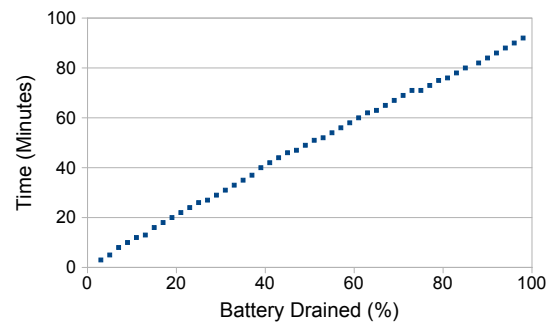

(f) Energy drain of most efficient attack over full battery life

Figure 4: Exploitation using software elements. 
pect that together they would drain five percent of the battery in under three minutes. Our test showed that the three components drained the battery in, on average, 4.8 minutes. This implies that there is some overlapping usage within groups of components. We also did a larger scale test of these three components. We started with a full battery and ran all three components. For this test we set a threshold of 2 percent, at which point the application wrote the battery level and total time to a file. How this attack drained the battery over time is shown by Figure $3(\mathrm{~b})$. The battery starts at 100 percent charge and drains down to 0 percent in just over 100 minutes by this attack. We also see that the attack drains almost linearly over time. This implies that the reduction in usage of some of the components at low power does not make a large impact on the overall attack. However, this did cause the device to become warm to the touch. Depending on the context of usage of the device, this may be difficult to detect or could be a major indicator of high battery usage.

We also did some tests with our normal 5 percent threshold while the device was connected and charging via USB. Despite the phone being charged while the attack was going on, the attack was still able to drain $5 \%$ of energy in an average of 7.2 minutes. This suggests that an energy attack is certainly able to kill the device even when it is charging. Moreover, Android allows any program to monitor the battery level and charging state without any extra permissions. An attacker could use this as a cue to scale up the energy attack when the device is charging to ensure that the device is killed.

Attack by taking photos drains the battery from $100 \%$ charge to $0 \%$ in about 265 minutes (Figure 3(c) . By just (stealthily) taking photos using the camera, an Android phone can be drained out of battery in over four and half hours (less than the flight time from New York to San Francisco).

\subsection{Software elements}

We carried out experiments demonstrating how attacks using encryption can be used to drain battery. In Figure 4(a), a $100 \%$ charged battery was drained fully about 200 minutes. However, the battery was about $95 \%$ drained at 175 minutes, and during the remaining 25 minutes it drained 5\% because Android reduced the brightness of the display due to its in-built policies.

In another exploit that implemented energy drain via repetitive database procedure: generation of strings of about $1 \mathrm{~KB}$, its addition to a table in the database and then deletion of the data, the battery was fully drained within 260 minutes (there is a similar spike towards the end due to savings of energy by dimming the display by Android) (Figure 4(b) . In Figure 4(c) the battery is drained fully within 300 minutes by addition and deletion of tables in a database on the device. In order to make the database-related exploit more effective, we included encryption in the exploit: data is generated, encrypted, added to the table, and then deleted. Figure 4(d) shows that encryption improved the exploit effectivenes by about considerable 20 minutes - it took about 240 minutes. We could add more cryptography operations to improve this period.

We also demonstrated how a web-app with a malicious javascript can carry out an attack. Figure 4(e) shows that such an attack is highly effective - it took only 164 minutes to drain the battery of the device fully. The javascript carries out network traffic and CPU usage. The attack showed no slow down at low power and stayed fairly consistent throughout the test. Every time the web application is killed it is brought back up, to the same web page, by our malicious application.

\subsection{Network Elements}

As described in the table above, Wifi-based data transfer drains $5 \%$ of energy at an average rate of 23.5 minutes. Bluetooth-based data transfer drains $5 \%$ energy at an average rate of 25.2 minutes. However, 4G-based data transfer consumes the most energy at an average of 11.1 minutes over 5\% drain. Due to space constraints, we could not add the plots related to the energy attacks due to network-based data transfer.

Most efficient attack: An exploit based on several hardware, software and network elements is developed. As Figure 4(f) shows this attack takes least amount of time -92 minutes to drain the battery from $100 \%$ to $0 \%$.

\subsection{Configuration of permissions}

Permissions are not needed for webpages to access default elements. However, for apps, permissions for each hardware element has to be allowed, so that a malicious energy attack app has to choose from those set of components it has the privilege of accessing. Generally the setting will have to be turned on by the user unless the application is given the required permission to change settings. The following table specifies the permissions required for each element. The final column marks whether the permission is required even if the required settings are enabled.

\begin{tabular}{|l|l|l|l|}
\hline Component & Setting & Permission & Req. \\
\hline Vibration & - & VIBRATE & $\mathrm{Y}$ \\
\hline CPU & - & - & $\mathrm{N}$ \\
\hline Camera Flash & - & FLASHLIGHT & $\mathrm{Y}$ \\
\hline WiFi Down. & WiFi enabled & $\begin{array}{l}\text { CHANGE } \\
\text { WIFI STATE }\end{array}$ & $\mathrm{N}$ \\
\hline Bluetooth & $\begin{array}{l}\text { Bluetooth } \\
\text { enabled }\end{array}$ & BLUETOOTH & $\mathrm{Y}$ \\
\hline Phone & - & CALL PHONE & $\mathrm{Y}$ \\
\hline 4G Down. & $\begin{array}{l}\text { Mobile data en- } \\
\text { abled }\end{array}$ & $\begin{array}{l}\text { CHANGE } \\
\text { NETWORK } \\
\text { STATE }\end{array}$ & $\mathrm{N}$ \\
\hline Brightness & - & - & $\mathrm{N}$ \\
\hline Video & - & - & $\mathrm{N}$ \\
\hline GPS & GPS enabled & $\begin{array}{l}\text { ACCESS FINE } \\
\text { LOCATION }\end{array}$ & $\mathrm{Y}$ \\
\hline Notification & - & - & $\mathrm{N}$ \\
\hline Rotation & - & - & $\mathrm{N}$ \\
\hline Photo & - & CAMERA & $\mathrm{Y}$ \\
\hline Encryption & - & - & $\mathrm{N}$ \\
\hline
\end{tabular}

Entries with '-' indicate that no setting or permission is needed. All of the permissions are under android.permission. All of the components with no required settings or permissions are available to any program under the Android API. For the case of normal use these permissions should give a good idea of how a desired application may impact battery usage. This information can be used to make good decisions about whether an application is a potential risk to battery life. These should also inform users on what they may need to monitor in each application to see if it is using excessive battery.

battery and their possibility of draining battery without the user knowing. Overall for normal usage the take-away is that it is easy and important to monitor component usage, and have an idea how an application may impact battery life. For an attack it is important to be prepared. Turning off any components that are not being used can help prevent usage by applications without specific permissions. Monitoring programs will help detect abnormal usage if an attack occurs. Disabling the camera, through administrative access, may be an important part of preventing an attack. It is im- 
portant to note that these can help mitigate the risk but will not prevent it. An application still has access to some resources without any permissions and many components may be hard to detect if the user does not know what they are looking for or does not recognize the change.

\section{RELATED WORK}

Malicious Battery Draining Apps. Even though, we are not aware of any malicious implementation of energy attacks for smartphones running iOS and Android, we have found apps that may carry out energy attacks inadvertently or as a side effect of other malicious activities such as data harvesting or a side effect of suboptimized code. Some of the apps we have found to be draining energy of a device by operations that are not needed:

- "Flashlight" [3] by Quick Switch on iPhone 4S: It acquires GPS, sends the location to the website perhaps for locationspecific ads and does not release it for sometime. It has been found to drain the battery of the phone overnight, if the app is not stopped.

- Waze [1] app does not release the "GPS" for several minutes even though the user has stopped using the app. That leads to battery drainage.

Energy Analysis on Mobile Devices Over the past decade, the battery usage analysis of mobile devices has attracted a lot of attentions. Neugebauer and McAuley [9] had suggested that using performance counter data to accurately account power consumption for laptops and mobile devices. To detect energy-greedy mobile malware such as WiFi faker, Kim et al. [4] proposed a power-aware malware detection framework by collecting application power consumption signatures. Recently, Carroll and Heiser [2] has systematically measured the energy usage and battery lifetime of a modern mobile device, at the major subsystem level such as graphics, GSM, WIFI, with a wide range of usage scenarios. EPROF [10] is an energy profiler that can quatitively measure the battery usage of smartphone apps, from system call tracing perspective based on their earlier research [11]. Similarly, AppScope [14], an Androidbased energy metering system, uses an event-driven kernel activity monitoring to meter the application energy usage. For troubleshooting battery drain issues, eDoctor [7] is a tool that can identify an abnormal app and suggest appropriate repair solution to users.

Compared to all these works, the substantial difference is we have different research goals. In particular, Kim et al. [4] focused on malware detection, Carroll and Heise [2] focused on identifying the critical components that consumes the most energy and then giving directions to power management, EPROF [10] and AppScope [14] focused on the profiling of energy usage of mobile apps, eDoctor [7] focused on the energy diagnosis, whereas we focus on identify the stealthy and promising approach to quickly drain the battery from offensive perspective. On the other hand, all these techniques can facilitate us for the better measurement of the energy usage for each specific attack.

Energy Analysis on Servers While energy attack has not been fully explored in the mobile devices, there are works that focus on the server side. Specifically, Wu et al. [13] studied the security aspect of modern computer power management, and designed an energy attack that exploits a standalone server system, in a similar fashion to the DoS attack. Not for offensive purposes, Li et al. [6] studied the power consumption on modern enterprise storage systems for a better power efficient design. In addition, for energy savings purposes, Sueur and Heiser [5] analyzed the modern energy saving techniques with various workloads and shed lights on how we should analyze the usage of power-management mechanisms.

\section{CONCLUSIONS}

Energy is a critical resource in mobile devices, and thus the emerging dependence on smartphones for critical tasks and activities is also tied to energy availability on the device. In this paper, we systematically analyze the attack vectors from hardware, software, and network communication perspective to drain the battery of a smartphone. In this paper, we have demonstrated that energy attack on smartphones is practical, and there are many incentives to carry out these attacks. We have studied the attack space, the taxonomy and the elements needed to build exploits for such attacks. We have also designed a number of concrete exploits, and our experimental results show how individual components as well as their combinations may be used to drain off the battery and mobile device users should be aware of these attacks.

\section{REFERENCES}

[1] Waze social gps maps and traffic. https://play.google.com/store/apps/details?id=com.waze.

[2] A. Carroll and G. Heiser. An analysis of power consumption in a smartphone. In Proceedings of the 2010 USENIX conference on USENIX annual technical conference, USENIXATC'10, pages 21-21, Berkeley, CA, USA, 2010. USENIX Association.

[3] D. Gilbert. First ios malware discovered in apple's app store, 2012. http://apple.slashdot.org/story/12/07/05/1727215/firstios-malware-discovered-in-apples-app-store.

[4] H. Kim, J. Smith, and K. G. Shin. Detecting energy-greedy anomalies and mobile malware variants. In Proceedings of the 6th international conference on Mobile systems, applications, and services, MobiSys '08, pages 239-252, New York, NY, USA, 2008. ACM.

[5] E. Le Sueur and G. Heiser. Slow down or sleep, that is the question. In Proceedings of the 2011 USENIX conference on USENIX annual technical conference, USENIXATC'11, pages 16-16, Berkeley, CA, USA, 2011. USENIX Association.

[6] Z. Li, K. M. Greenan, A. W. Leung, and E. Zadok. Power consumption in enterprise-scale backup storage systems. In Proceedings of the 10th USENIX conference on File and Storage Technologies, FAST'12, pages 6-6, Berkeley, CA, USA, 2012. USENIX Association.

[7] X. Ma, P. Huang, X. Jin, P. Wang, S. Park, D. Shen, Y. Zhou, L. K. Saul, and G. M. Voelker. edoctor: automatically diagnosing abnormal battery drain issues on smartphones. In Proceedings of the 10th USENIX conference on Networked Systems Design and Implementation, nsdi'13, pages 57-70, Berkeley, CA, USA, 2013. USENIX Association.

[8] D. Murphy. Industrious users performing imessage denial-of-service attacks, 2013. http://www.pcmag.com/article2/0,2817,2417267,00.asp.

[9] R. Neugebauer and D. McAuley. Energy is just another resource: Energy accounting and energy pricing in the nemesis os. In Proceedings of the Eighth Workshop on Hot Topics in Operating Systems, HOTOS '01, pages 67-, Washington, DC, USA, 2001. IEEE Computer Society.

[10] A. Pathak, Y. C. Hu, and M. Zhang. Where is the energy spent inside my app?: fine grained energy accounting on smartphones with eprof. In Proceedings of the 7th ACM european conference on Computer Systems, pages 29-42. ACM, 2012. 
[11] A. Pathak, Y. C. Hu, M. Zhang, P. Bahl, and Y.-M. Wang. Fine-grained power modeling for smartphones using system call tracing. In Proceedings of the sixth conference on Computer systems, EuroSys '11, pages 153-168, New York, NY, USA, 2011. ACM.

[12] K. Paul and T. Kundu. Android on mobile devices: An energy perspective. In Computer and Information Technology (CIT), 2010 IEEE 10th International Conference on, pages 2421-2426, 2010.

[13] Z. Wu, M. Xie, and H. Wang. Energy attack on server systems. In Proceedings of the 5th USENIX conference on Offensive technologies. USENIX Association, 2011.

[14] C. Yoon, D. Kim, W. Jung, C. Kang, and H. Cha. Appscope: application energy metering framework for android smartphones using kernel activity monitoring. In Proceedings of the 2012 USENIX conference on Annual Technical Conference, USENIX ATC'12, pages 36-36, Berkeley, CA, USA, 2012. USENIX Association.

[15] Y. Zhou and X. Jiang. Dissecting android malware: Characterization and evolution. In Security and Privacy (SP), 2012 IEEE Symposium on, pages 95-109. IEEE, 2012. 